Why misinformation is more likely to be recognised over time:

A source monitoring account

\author{
Peter Frost \\ Southern New Hampshire University \\ Melissa Ingraham and Beth Wilson \\ Rivier College
}

Citation: Frost, P., Ingraham, M., \& Wilson, B. (2002). Why misinformation is more likely to be recognised over time: A source monitoring account. Memory, 10(3), 179-185. doi:10.1080/09658210143000317.

Submitted: 5 March 2001. Accepted: 30 August 2001 
Why misinformation is more likely to be recognised over time 2

\begin{abstract}
Although memory for actual events tends to be forgotten over time, memory for misinformation tends to be retrieved at a stable rate over long delays or at a rate greater than that found immediately after encoding. To examine whether source monitoring errors contribute to this phenomenon, two experiments investigated subjects' memory for the source of misinformation at different retention intervals. Subjects viewed a slide presentation, read a narrative containing misinformation, and, either 10 minutes or 1 week later, completed a recognition test about details seen in the slides and about the source of these details. After the longer retention interval in both experiments, participants were more likely to agree that they had seen misleading information and were also more likely to incorrectly associate the misinformation with the slide event. Theoretical implications of these findings are considered.
\end{abstract}


Why misinformation is more likely to be recognised over time 3

\section{Why misinformation is more likely to be recognised over time: A source monitoring account}

Researchers investigating eyewitness memory have long found that participants retrieve poste-vent misinformation, even when asked to remember only what was seen during an eye-witness episode (Belli, 1989; Frost \& Weaver, 1997; Loftus, Miller, \& Burns, 1978; McCloskey\& Zaragoza, 1985; Weingart, Loftus, \& Lindsay, 1995). What makes this "misinformation effect" even more problematic is that recent evidence suggests that memory for misinformation appears to be more resistant to forgetting than memory for actual details of an eyewitness event. Some research has shown that, after relatively long delays, misinformation tends to be recalled and recognised at a rate similar or greater than immediately after exposure to misinformation (Frost, 2000; Underwood \& Pezdek, 1998). Underwood and Pezdek (1998), for instance, found that participants tend to recognise more misinformation after a 1-month retention interval under certain conditions. Participants first viewed slides - the simulated eyewitness event - depicting a young woman baking a cake, and another set of slides depicting a man surveying a construction site. Participants then read two narratives, each describing one of the slide sets. Each narrative contained misinformation that contradicted details seen in the slides. Participants were told that the narrative was either attributed to a low-credibility source - a 4year-old boy - or a high-credibility source - a memory psychologist. Participants then answered a yes/no recognition test about details in the slides. After a 1-month delay between the time of reading the narrative and test, participants tended to recognise more mis-information than after a 10-minute delay, especially in the low-credibility condition.

To explain this finding, Underwood and Pezdek apply a hypothesis associated with communication effectiveness, called the availability-valence hypothesis. According to this hypothesis, people tend to rate the message from a low-credibility source as more effective over time because the message and its source become less strongly associated after long delays (Hoveland, Lumsdaine, \& Sheffield, 1949; Mazursky \& Shul, 1987). At short delays however, participants remember the low-credibility source is associated with the message (or misinformation), so they are hesitant to judge the message as effective. Applied to the misinformation paradigm, this term could be taken to mean that people will tend to falsely recognise misinformation as true over long peri-ods of time. The underlying assumption is that, although misinformation may remain familiar over time - enough to be recognised - memory for 
Why misinformation is more likely to be recognised over time 4

the source of the misinformation will deteriorate to the extent that a participant will more likely accept it as having been from the eyewitness event.

The availability-valence hypothesis is a variant of what is more generally referred to as source monitoring errors (Johnson, Hashtroudi, \& Lindsay, 1993; Lindsay, 1990). According to this account, every memory, including memory for misinformation, has a source that is decided upon by the witness at the time of retrieval. Misinformation is sometimes recognised because the source of misinformation is confused as being from the eyewitness event (Belli, Lindsay, Gales, \& McCarthy, 1994; Zaragoza \& Lane, 1994).

Furthermore, Johnson et al. (1993) claim that the more characteristics the memory for misinformation has in common with the memory for the event, the more likely it is the participant will confuse the source of misinformation as being from the event. Retention time, for instance, will play an important role in how similar the details of a memory for misinformation will be relative to the memory for the event. Over a short delay, it is more obvious that the memory for the verbal misinformation does not have some of the perceptual characteristics of the pictorial eyewitness event, leading to a greater likelihood that the participant will not accept misinformation as being from the event. However, as the memory for the pictorial event loses its perceptual details over time, it becomes more comparable to the memory of misinformation in regard to the quantity of details associated with it, making source mon-itoring errors more likely. To become more comparable with real memory, it is unlikely that memory for misinformation gains more perceptual details over time; misled and real memories are eventually more likely to be confused because of a mutual lack of details that arise as time progresses. After a long delay, the source mis-attribution account predicts that participants would be less hesitant to recognize misinformation as being from the event, thus offering a possible account for the increased misinformation effects over time found in Frost (2000) and Underwood and Pezdek (1998).

As predicted by the source monitoring account, studies that have included a relatively short delay have shown that participants tend to correctly attribute misinformation to the narrative rather than to an event depicted on slides (Lindsay \& Johnson, 1989; Zaragoza \& Koshmider, 1989). If, however, participants are encouraged to mentally reconstruct the original event or visualise misleading details - thereby adding visual details to the misinformation source monitoring errors become more likely, even after relatively short delays (Durso \& Johnson, 1980; Zaragoza \& Lane, 1994). 
Why misinformation is more likely to be recognised over time 5

To our knowledge, no one has assessed memory for source in a misinformation paradigm while varying retention time within a single experiment. If source monitoring errors contribute to increased misinformation effects over time, then memory for source should be particularly poor for misled memory after a long delay while mis-information recognition performance remains steady or increases over the interval. In the following two experiments, recognition memory for misinformation and its source was measured at different retention intervals (10 minutes and 1 week).

\section{Experiments 1 and 2}

As Experiments 1 and 2 involved virtually the same procedures, the methodology and results of these experiments will be described together. The only difference between experiments was that participants in Experiment 2 were told that some information read in a narrative (containing misinformation) was inconsistent with what was seen in a slide presentation, whereas participants in Experiment 1 did not receive this instruction. This variation in procedure was included to avoid demand characteristics. For instance, participants might appear to misattribute misleading details to the eyewitness event not because of source monitoring confusion, but because participants trusted that misleading details read about in the narrative were in the original event. Although they might know that misleading details were from the narrative, they infer that the narrative contained correct information about the event. By instructing participants that some of the information in the narrative was inconsistent with the event, the tendency to assume narrative items were in the event is avoided. This approach has been referred to as the logic of opposites (Lindsay, 1990).

\section{Method}

Participants. Each experiment included 48 undergraduate students from Rivier College in Nashua, New Hampshire. Participants were offered extra credit to volunteer in the experiments. Participants were randomly assigned to a 10-minute or 1-week delay condition, with 24 participants in each condition.

Materials. A total of 46 slides were presented (a subset from McCloskey \& Zaragoza, 1985). These slides portray an incident in an office where a maintenance man steals 20 dollars and a calculator. Four details, each seen in a separate slide, were critical details. One of two 
Why misinformation is more likely to be recognised over time 6

versions of each critical detail was presented at a particular session: An initial on a mug ("L" or “M”), a soft-drink can (Coca-Cola or Sunkist), a magazine (Glamour or Vogue), and a tool (hammer or wrench). During each experiment session, two control critical details were not later associated with misinformation while the two remaining critical details were associated with misinformation in a subsequent narrative. Each version of the critical details was counterbalanced in such a way as to ensure that each version served as control details and details associated with misinformation an equal number of times.

Participants read a narrative describing two misinformation details that contradicted two cri-tical details seen in the slides. For instance, the narrative might mention that the maintenance man pulled out a hammer (misinformation detail) from the toolbox, when, actually, a wrench (critical detail) was shown in the slides. The narrative included 26 sentences detailing the incident in the slides.

A yes/no test similar to that used by Underwood and Pezdek (1998) was administered after the narrative. Each of the four critical details was assessed by three yes/no questions, referring to the slide details, misinformation details, and foil details. Serving as a baseline, foil details were those details that were neither in the slide nor the narrative. Participants were asked to circle yes or no to questions that took the following form: "Did the man place the keys next to a Coca-Cola can, yes or no?" Participants were asked not to circle yes or no if they did not remember the answer.

Each question about slide, misleading, and foil items was also followed by two questions about the source of the detail. Questions took the fol-lowing form: "Did you see the Coca-Cola can in the slide event, yes or no?" Another question asked, "Did you see the Coca-Cola can in the narrative, yes or no?" Again, participants were instructed not to circle yes or no if they did not know the answer.

Procedure. Participants viewed slides at a rate of 5 seconds each. After the slide presentation was completed, participants worked on a crossword puzzle, used as a filler task, for 5 minutes. They then read the narrative at their own pace. After the narrative, participants completed the yes/no recognition test either 10 minutes or 1 week after they had started reading the narrative. Retention interval was varied between subjects. After participants in the 10-minute condition finished reading the narrative, they were again provided with a filler task (crossword puzzle). After participants in the 1-week condition finished reading the narrative, they were 
asked not to discuss the experiments with anyone until the next experimental session. After the 10-minute or 1-week delay, participants were given the yes/no recognition test with instructions to circle yes for details recognized from the slides. In regard to answering questions about the source of the items, participants were told that they could indicate that details were seen in both the slides and the narrative. In Experiment 2, participants were informed just before administration of the test that some information in the narrative was inconsistent with details shown in the slides. When the experiment was completed, participants were debriefed.

TABLE 1

Proportion of "yes" responses to details as a function of experiment, retention interval, and condition

\begin{tabular}{|c|c|c|c|}
\hline \multirow[b]{2}{*}{$\begin{array}{l}\text { Delay/ } \\
\text { Condition }\end{array}$} & \multicolumn{3}{|c|}{ Question type } \\
\hline & $\begin{array}{c}\text { Misleading } \\
\text { details }\end{array}$ & $\begin{array}{c}\text { Slide } \\
\text { details }\end{array}$ & $\begin{array}{c}\text { Foil } \\
\text { detail }\end{array}$ \\
\hline \multicolumn{4}{|c|}{ Experiment 1} \\
\hline \multicolumn{4}{|c|}{ 10-Minutes } \\
\hline Control & .04 & .70 & .09 \\
\hline Misled & .30 & .46 & .13 \\
\hline \multicolumn{4}{|l|}{ 1-Week } \\
\hline Control & .04 & .58 & .13 \\
\hline Misled & .38 & .33 & .16 \\
\hline \multicolumn{4}{|c|}{ Experiment 2} \\
\hline \multicolumn{4}{|c|}{ 10-Minutes } \\
\hline Control & .02 & .64 & .04 \\
\hline Misled & .25 & .45 & .05 \\
\hline \multicolumn{4}{|l|}{ 1-Week } \\
\hline Control & .00 & .55 & .07 \\
\hline Misled & .35 & .28 & .07 \\
\hline
\end{tabular}

10-minutes and 1-week refer to the retention times between the start of the narrative phase and the start of the recognition test phase. 
Why misinformation is more likely to be recognised over time 8

\section{Results and discussion}

Table 1 shows the proportion of "yes" responses to the question types (i.e., questions about mis-leading, slide, and foil details) as a function of Experiment (Experiments 1 and 2), retention interval (10 minutes and 1 week), and condition (misled and control). The effects of each of the three details were analysed separately, using a 2 (retention interval: 10 min or 1 day) x2 (condition: misled or control) mixed factorial analysis of variance (ANOVA). ANOVAs were conducted for Experiment $1(F 1)$ and for Experiment $2(F 2)$. All analyses in both experiments were set at alpha equal to .05 .

Participants' responses to misleading details across condition and retention interval were analysed. Participants were more likely to recognise misleading details in the misled condition than in the control condition. ${ }^{1}$ The main effect of condition was significant, $F 1(1,44)=13.54$, $\mathrm{MSE}=2.24$ and $F 2(1,44)=19.29, \mathrm{MSE}=3.13$. The main effect of retention interval was also significant, $F 1(1,44)=4.86, \mathrm{MSE}=2.24$ and $F 2(1,44)=6.16, \mathrm{MSE}=3.13$, with more misleading details being recognised over the delay. An interaction between condition and retention interval did not occur, $F 1(1,44)=0.25, \mathrm{MSE}=2.24$ and $F 2(1,44)=0.15, \mathrm{MSE}=3.13$.

Slide details and foil details were also analysed separately using a 2 (retention interval) $\mathrm{x}$ 2 (condition) mixed ANOVA. For slide details, only the main effects for condition, $F 1(1,44)=$ 11.86, $\mathrm{MSE}=2.23$ and $F 2(1,44)=9.89, \mathrm{MSE}=2.73$, and retention interval, $F 1(1,44)=9.99$, $\mathrm{MSE}=2.23$ and $F 2(1,44)=8.39, \mathrm{MSE}=2.73$, were significant. An interaction between condition and retention interval did not occur when slide details were analysed, $F 1(1,44)=0.77$, $\mathrm{MSE}=2.23$ and $F 2(1,44)=0.57, \mathrm{MSE}=2.73$. For foil details, no main effects were found for condition, $F 1(1,44)=0.21, \mathrm{MSE}=1.86$ and $F 2(1,44)=0.13, \mathrm{MSE}=1.08$, nor for time, $F 1(1,44)$ $=0.75, \mathrm{MSE}=1.86$ and $F 2(1,44)=1.08, \mathrm{MSE}=1.08$. An interaction between condition and retention interval did not occur when foil items were analysed, $F 1(1,44)=0.14$, MSE $=1.86$ and $F 2(1,44)=0.18, \operatorname{MSE}=1.08$.

\footnotetext{
${ }^{1}$ A misleading detail in the control condition referred to a detail that would have been
} used to contradict the critical detail as misinformation in other conditions. Retrieval of these items served as a baseline. 
Why misinformation is more likely to be recognised over time 9

To examine whether the resulting misinformation effect was due to guessing and whether there was an increase in the recognition of misleading details relative to foil details over time, the pro-portion of yes responses to misleading and foil details was compared in the misled condition after the different delays using a 2 (question type: misleading or foil detail) x 2 (time: 10 minutes or 1 week) mixed ANOVA. Considering details in the misled conditions, the main effect for question type was significant, with the proportion of recognized foil details (.15 in Experiment 1 and .12 in Experiment 2) significantly lower than the proportion of recognized misleading details $(.34$ in Experiment 1 and .30 in Experiment 2$), F 1(1,44)=26.45, \mathrm{MSE}=1.04$ and $F 2(1,44)=$ 42.45, $\mathrm{MSE}=1.82$. Therefore, misinformation was recognized at a rate greater than the recognition rate associated with mere guessing. An interaction between question type and retention interval in the misled conditions was also significant, $F 1(1,44)=2.61$, MSE $=1.04$ and $F 2(1,44)=11.05, \mathrm{MSE}=1.82$. While the proportion of misleading details recognised increased over the retention interval, the proportion of foil details recognised did not increase over the retention interval.

Table 2 shows the proportion of yes responses to the details and the potential sources of those details, broken down by experiment, retention interval, and condition. Participants tended to commit more source monitoring errors for both misleading and slide details over time. A main effect for time was found when misleading details in the misled conditions were misattributed to the slides, $F 1(1,44)=19.43, \mathrm{MSE}=2.18$ and $F 2(1,44)=11.22, \mathrm{MSE}=2.74$. A main effect for time was also found when slide details in the control conditions were misattributed to the narrative, $F 1(1,44)=34.75, \mathrm{MSE}=2.07$ and $F 2(1,44)=29.83, \mathrm{MSE}=2.51$.

In regards to misleading details, participants tended to correctly attribute misinformation to the narrative after a short delay. Notice that when misleading details were recognised in the 10-minute, misled condition, participants correctly indicated more often that these details came from the narrative rather than the slides, especially when they were told about the inconsistent details in Experiment 2. After a week, however, participants in the misled condition were more likely to incorrectly indicate that misleading details were from the slide rather than the narrative. A different pattern emerged for slide details over time. In the 10-minute, control condition, more slides details were indicated as belonging to the slides than to the narrative. While memory for the source of the slides also diminished over time, participants were still more likely to correctly 
TABLE 2

Proportion of "yes" responses to details (overall) and source of details as a function of experiment, retention interval, and condition

\section{Question type}

Misleading details

\begin{tabular}{lcccccc} 
Delay/Condition & Overall & Slide? & Narrative? & Overall & Slide? & Narrative? \\
\hline $\begin{array}{l}\text { Experiment } 1 \\
\text { 10-Minutes }\end{array}$ & & & & & & \\
Control & .04 & .02 & .02 & .70 & .69 & .03 \\
Misled & .30 & .11 & .19 & .46 & .44 & .06 \\
& & & & & & \\
1-Week & .04 & .03 & .03 & .58 & .37 & .30 \\
Control & .38 & .25 & .16 & .33 & .23 & .18 \\
Misled & & & & & &
\end{tabular}

Experiment 2

10-Minutes

$\begin{array}{lllllll}\text { Control } & .02 & .02 & .00 & .64 & .64 & .00 \\ \text { Misled } & .25 & .08 & .17 & .45 & .43 & .02\end{array}$

1 -Week

$\begin{array}{lllllll}\text { Control } & .00 & .00 & .00 & .55 & .35 & .22 \\ \text { Misled } & .35 & .22 & .17 & .28 & .18 & .14\end{array}$

In some instances the sum of the proportion of yes responses to slide and narrative sources does not equal the overall proportion. This is because subjects could - and in some cases did - say yes to both slide and narrative sources.

attribute the slide details to the slides than to the narrative after a 1-week interval. Source mon-itoring errors were less likely to occur for slide details after a delay than for misleading details.

\section{General Discussion}

These experiments replicate the finding that memory for misinformation tends to be retrieved with greater frequency over time than memory for actual event details (Frost, 2000; Underwood \& Pezdek,1998). The findings of these experiments also revealed that participants committed more source monitoring errors relative to correct source attributions for misleading 
Why misinformation is more likely to be recognised over time 11

details after a 1-week retention interval, whereas participants were more likely to correctly attribute slide details to the slides at both short and long intervals.

In Experiment 2, participants were told that there was some information in the narrative that was inconsistent with details seen in the slides. Despite this warning, participants still tended to misattribute misleading details to the slides more often than they correctly attributed the mis-information to the narrative after the 1-week delay. Because participants knew about the inconsistencies in the narrative, it is unlikely that participants merely appeared to misattribute misleading details to the slides because they inferred that the narrative was an entirely accu-rate account of what occurred in the slides. More likely, participants experienced genuine source confusions.

Consistent with Lindsay and Johnson (1989) and Zaragoza and Koshmider (1989), participants were more likely to correctly attribute misinformation to the narrative after a short delay. Control details also tended to be correctly attributed to their source, the slides, after a short delay.

The tendency for participants to correctly identify the source of misinformation and actual event details after a short delay was consistent with source monitoring accounts. The availability-valence account would predict that, after a brief delay, participants would still have in mind a strong association between the details seen and read and their sources, preventing source confusions. The findings over the short delay are also consistent with predictions made by Johnson et al. (1993), especially as the recent encoding of the event would maintain much of its perceptual detail in memory. The newly encoded verbal misinformation would lack the perceptual details of the event, thus preventing the participant from being inclined to recognize the misinformation as being from the slides.

The source monitoring error rates for misled and true memories tended to differ after a longer retention interval. After the long delay, participants were more likely to incorrectly attribute misinformation to the slides, whereas participants continued to correctly attribute slide details to the slides after a long delay, although to a lesser extent than after the short delay.

The finding that participants were more likely to confuse misinformation as belonging to the eyewitness event over time is consistent with source monitoring accounts. According to the availability-valence hypothesis, it is after the long retention interval that the association between the items and their sources breaks down, and participants are more likely to confuse 
Why misinformation is more likely to be recognised over time 12

misinformation as having been seen in the slides. This finding is also consistent with the predictions of Johnson et al. (1993) because it is after a delay that memory for misinformation becomes more comparable to the event memory in terms of the quantity of perceptual details, making source monitoring errors of misinformation to the slides more likely.

What source monitoring hypotheses do not account for, however, is the fact that, after a relatively long delay, the majority of source attributions to misinformation were incorrect, whereas the majority of source attributions to event details were correct. Is this because participants are more motivated to attribute details to the eyewitness event, even after the memory for source becomes vague? Or is it because memory for misinformation is truly more susceptible to source confusions than memory for actual event details? Future research will need to examine the mechanism(s) behind this particular finding.

Source monitoring accounts do offer reasons why people are more inclined to retrieve misinformation over time. After a delay, more misinformation is likely selected because the source of misinformation becomes lost in memory in many cases. Misinformation that the eyewitness initially hesitated to recognise remains familiar after a long delay, but loses its association with its source and is more comparable to the eyewitness episode in terms of its lack of details.

\section{Conclusion and implications}

Although past studies have not specifically focused on the phenomenon of increased recognition of misled memories over time, this study, taken together with the results of Frost (2000) and Underwood and Pezdek (1998), suggests that this is a fairly robust phenomenon worthy of more attention. It is also clear that source monitoring errors, more frequent after relatively long retention intervals, may be linked to an increase in the recognition of misinformation overtime. Investigations can now focus on why memory for misinformation is especially susceptible to source confusions compared with accurate memories after a delay. Given the abundance of information people encounter today, it is important to confirm the legitimacy of the source of information when it is based on long-term memory. 
Why misinformation is more likely to be recognised over time 13

\section{References}

Belli, R.F. (1989). Influences of misleading postevent information: Misinformation interference and acceptance. Journal of Experimental Psychology: General, 118, 72-85.

Belli, R.F., Lindsay, D.S., Gales, M.S., \& McCarthy, T.T. (1994). Memory impairment and source mis-attribution in postevent misinformation experiments with short retention intervals. Memory and Cognition, 22(1), 40-54.

Durso, F.T., \& Johnson, M.K. (1980). The effects of orienting tasks on recognition, recall, and modality confusion of pictures and words. Journal of Verbal Learning and Verbal Behavior, 19, 416-429.

Frost, P.J. (2000).The quality of false memory over time: Is memory for misinformation "Remembered" or "Known"? Psychonomic Bulletin and Review, 7(3), 531-536.

Frost, P.J., \& Weaver, C.A. III (1997). Overcoming misinformation effects in eyewitness memory: Effects of encoding time and event cues. Memory, 5(6), 725-740.

Hovland, C.I., Lumsdaine, A.A., \& Sheffield, F.D. (1949). Experiments in mass communication. Princeton, NJ: Princeton University Press.

Johnson, M.K., Hashtroudi, S., \& Lindsay, D.S.(1993). Source monitoring. Psychological Bulletin, 114, 3-28.

Lindsay, D.S. (1990). Misleading suggestions can impair eyewitnesses' ability to remember event details. Journal of Experimental Psychology: Learning, Memory, and Cognition, 16(6), 1077-1083.

Lindsay, D. S., \& Johnson, M.K. (1989). The eyewitness suggestibility effect and memory for source. Memory and Cognition, 17, 349-358.

Loftus, E.F., Miller, D.G., \& Burns, H.J. (1978). Semantic integration of verbal information into a visual memory. Journal of Experimental Psychology: Human Learning and Memory, 4, 19-31.

Mazursky, D., \& Shul, Y. (1987). The effects of advertisement encoding on the failure to discount information: Implications for the sleeper effect. Journal of Consumer Research, 15, 24 35.

McCloskey, M., \& Zaragoza, M. (1985). Misleading postevent information and memory for events: Arguments and evidence against memory impairment hypotheses. Journal of Experimental Psychology: General, 114, 1-16. 
Underwood, J., \& Pezdek, K. (1998). Memory suggestibility as an example of the sleeper effect. Psychonomic Bulletin and Review, 5, 449-453.

Weingardt, K.R., Loftus, E.F., \& Lindsay, D.S. (1995). Misinformation revisited: New evidence on the suggestibility of memory. Memory and Cognition, 23(1), 72-82.

Zaragoza, M.S.,\& Koshmider, J.W. III (1989). Misled subjects may know more than their performance implies. Journal of Experimental Psychology: Learning, Memory, and Cognition, 15(2), 246-255.

Zaragoza, M.S., \& Lane, S.M. (1994). Source misattribution and the suggestibility of eyewitness memory. Journal of Experimental Psychology: Learning, Memory, and Cognition, 20(4), 934-945. 